\title{
Advanced low-beta cavity development for proton and ion accelerators
}

\author{
Z.A. Conway ${ }^{\mathrm{a} *}$, M.P. Kelly ${ }^{\mathrm{a}}$ and P.N. Ostroumov ${ }^{\mathrm{a}}$ \\ ${ }^{a}$ Argonne National Laboratory, 9700 S. Cass Ave., Argonne, Illinois 60439
}

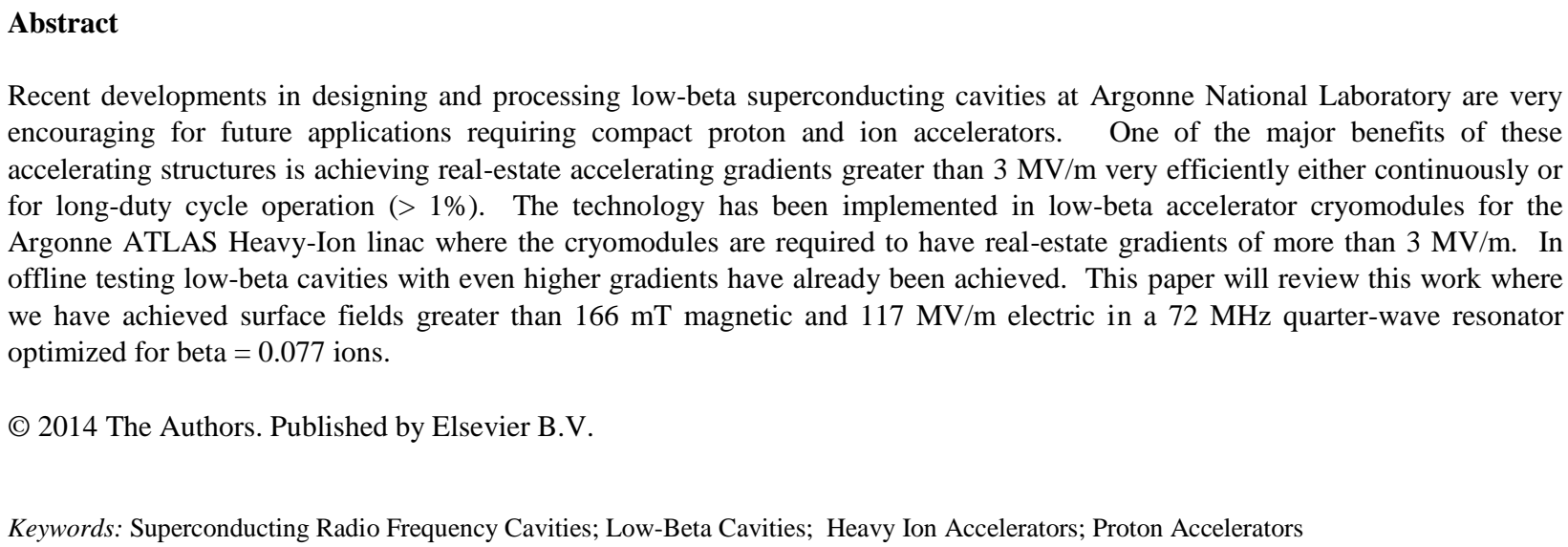
encouraging for future applications requiring compact proton and ion accelerators. One of the major benefits of these accelerating structures is achieving real-estate accelerating gradients greater than $3 \mathrm{MV} / \mathrm{m}$ very efficiently either continuously or for long-duty cycle operation $(>1 \%)$. The technology has been implemented in low-beta accelerator cryomodules for the Argonne ATLAS Heavy-Ion linac where the cryomodules are required to have real-estate gradients of more than $3 \mathrm{MV} / \mathrm{m}$. In offline testing low-beta cavities with even higher gradients have already been achieved. This paper will review this work where we have achieved surface fields greater than $166 \mathrm{mT}$ magnetic and $117 \mathrm{MV} / \mathrm{m}$ electric in a $72 \mathrm{MHz}$ quarter-wave resonator optimized for beta $=0.077$ ions.

(C) 2014 The Authors. Published by Elsevier B.V.

Keywords: Superconducting Radio Frequency Cavities; Low-Beta Cavities; Heavy Ion Accelerators; Proton Accelerators

\section{Main text}

After four decades of development, low-beta superconducting radio-frequency (SRF) accelerator cavities still do not routinely perform at the same peak-surface fields or quality factors which are regularly achieved in off-line tests of elliptical-cell cavities optimized for velocity-of-light electrons. Elliptical-cell cavities fabricated for the ILC and other projects have regularly achieved $160 \mathrm{mT}$ and $80 \mathrm{MV} / \mathrm{m}$ peak surface magnetic and electric fields respectively with RF surface resistances of $10 \mathrm{n} \Omega$ or less for several years with the proper fabrication and processing [1]. Lowbeta cavities $(\beta=\mathrm{v} / \mathrm{c}<0.5)$ are based on singly or multiply loaded cavity resonators where each loading element supports a TEM-like mode and recently several SRF cavities of this class have operated with surface magnetic fields $>120 \mathrm{mT}$ but with RF surface resistances greater than $10 \mathrm{n} \Omega[2,3]$. Even so, SRF cavities optimized for low-beta are widely used in existing ion-accelerators (e.g., ATLAS at Argonne National Laboratory [4] and ISAC-II at TRIUMF [5]) and are an integral part of several new low-beta linac proposals which require high-duty-factor or continuous wave operation and utilize technologies developed for higher velocity cavities $[6,7,8,9,10,11,12,13]$. In the operating regime required for all of these accelerators, superconducting cavities reduce the required power by 10-100 relative to normal conducting copper cavities and enable higher accelerating gradients with duty factors up to $100 \%$, hence, shortening the accelerator, reducing project costs and enabling higher duty factor operation.

The length and cost of the low-beta portion of superconducting proton and heavy-ion linear accelerators is dominated by the SRF accelerator cavity performance and improving these cavities to the level of those achieved by the ILC project is an important cost savings measure [14]. High performance accelerator cavities reduce: the size of

\footnotetext{
* Corresponding author. Tel.: 1-630-252-0874; fax: 1-630-252-9647.

E-mail address: zconway@anl.gov
} 
the cryogenic plant required for operation, the number of accelerator cavities and cryomodules required, the size of the RF power amplifiers, etc. Low-beta accelerator cavity performance has been improving over the past decade $[15,16,17]$ but much remains to be done to regularly achieve higher performance levels. The performance disparity relative to elliptical-cell cavities has been blamed on the greater complexity of the cavity fabrication and processing. Several advances at Argonne National Laboratory in cavity design, fabrication and processing [18, 19] have disproven this hypothesis and in this paper we present our results of a high-performance quarter-wave cavity fabricated and processed using many of the same techniques developed for elliptical cell cavities with correspondingly good results.

First, the results locating a defect which limited the performance of a prototype $72 \mathrm{MHz}$ quarter-wave cavity (QWR) optimized for $\beta=0.077$ ions for the ATLAS intensity upgrade will be presented to give an example of what limits cavity performance. Second, the fabrication, processing and testing of a subsequent geometrically identical quarter-wave cavity will be outlined where we focused on preventing cavity performance limiting defects. Third, a brief overview of the online performance of a cryomodule comprised of 7 of these quarter-wave cavities will be mentioned. Finally, the impact of these results and future plans will be discussed.

\section{Prototype 72 MHz Quarter-Wave Cavity}

The QWR prototype fabrication and test results were presented in [18]. Since this paper was published we have refined our electromagnetic simulations of the cavity surface fields and the results used in this paper are given in table 1. This cavity was limited to peak surface fields of $96 \mathrm{mT}$ and $70 \mathrm{MV} / \mathrm{m}$ by a surface defect which initiated a cavity quench.

The defect was located by measuring the time-of-flight of second sound waves propagating from the cavity quenching defect to an array of oscillating superleak transducers [20]. Figure 1 shows the cavity with the location of the defect highlighted in red along with a single channel record of the quench. The distance the second sound wave travelled was calculated with the second sound wave velocity at the bath temperature. This combined with the known location of the detectors allowed us to determine the defect location. Our detector array was 1-dimensional in nature and located the height of the defect in the quarter-wave cavity center conductor.

This defect was located at the same height as an electron beam weld blow-out which occurred during final welding of the center conductor. The center conductor is formed from two halves which are welded together. The center conductor halves were tacked welded together but the final electron beam welds could not be finished within the 24-hour etch window desired between pre-weld etching and welding. The parts were etched again after tacking and the very small-gap joint between the two parts could not be properly cleaned. Some foreign debris was trapped in this joint which caused a blow-out during the final electron beam welding which was repaired by filling the hole with a high-RRR niobium ball and welding over the blow-out again.

Table 1. Electromagnetic Properties of the QWR

\begin{tabular}{ll}
\hline Parameter & Value \\
\hline Frequency & $72.75 \mathrm{MHz}$ \\
$l_{\text {eff }}(\beta \lambda)$ & $31.75 \mathrm{~cm}$ \\
$\mathrm{E}_{\mathrm{pk}} / \mathrm{E}_{\mathrm{acc}}$ & 5.0 \\
$\mathrm{~B}_{\mathrm{pk}} / \mathrm{E}_{\mathrm{acc}}(\mathrm{mT} /(\mathrm{MV} / \mathrm{m}))$ & 7.1 \\
$\mathrm{QR}_{\mathrm{s}}(\Omega)$ & 25.9 \\
$\mathrm{R}_{\mathrm{sh}} / \mathrm{Q}(\Omega)$ & 568 \\
\hline
\end{tabular}




\section{Improved QWR Performance}

\subsection{Fabrication and Processing}

Here the fabrication details which differ from what was presented in [18] for the prototype QWR are listed:

- Very careful attention was paid to the status of the weld joints and their preparation prior to electron beam welding. All niobium components were cleaned and soaked in high-purity water after every handling/machining step to inspect for inclusions.

- The surface inspection and quality control were increased and improved. The cavity RF surface was carefully hand-polished with 220 grit sandpaper to avoid fold-over and deep polishing marks. All pits, orange-peel and scratches were polished in this manner in the high field regions.

- The temperature of the pre-weld etches were limited to $\mathrm{T}<16^{\circ} \mathrm{C}$ to limit hydrogen uptake. The bulk electropolish procedure $(\sim 120 \mu \mathrm{m}$ removal $)$ temperature was reduced to $25<\mathrm{T}<30^{\circ} \mathrm{C}$. The final light polish $(\sim 20 \mu \mathrm{m}$ removal $)$ temperature was also reduced to $20<\mathrm{T}<25^{\circ} \mathrm{C}$.

- The cavity was baked at $625^{\circ} \mathrm{C}$ for 24 hours to degas the hydrogen dissolved in the bulk niobium. This step was performed after the heavy electropolish but before the final light polish. This prevents Q-disease and improves the $2 \mathrm{~K}$ performance of the cavity.

Using these modifications we made an additional QWR, henceforth referred to as the DTRA QWR. After the metal fabrication for the DTRA QWR was complete the electropolishing, baking and cleaning proceeded. To maintain the temperature below $25^{\circ} \mathrm{C}$ during the final light electropolish the voltage had to be turned off and on several times to allow for the cavity and electrolyte to cool down; the polishing voltage was off for $\sim 3$ minutes and on for 10 minutes throughout the duration of the 40 minute procedure. Figure 2 shows the cavity temperature and a few select residual gas species during the hydrogen degassing bake and all numbers indicate that the bake was good. The cavity bake was done by Fermilab's Technical division and followed the traditional parameters for hydrogen degassing.
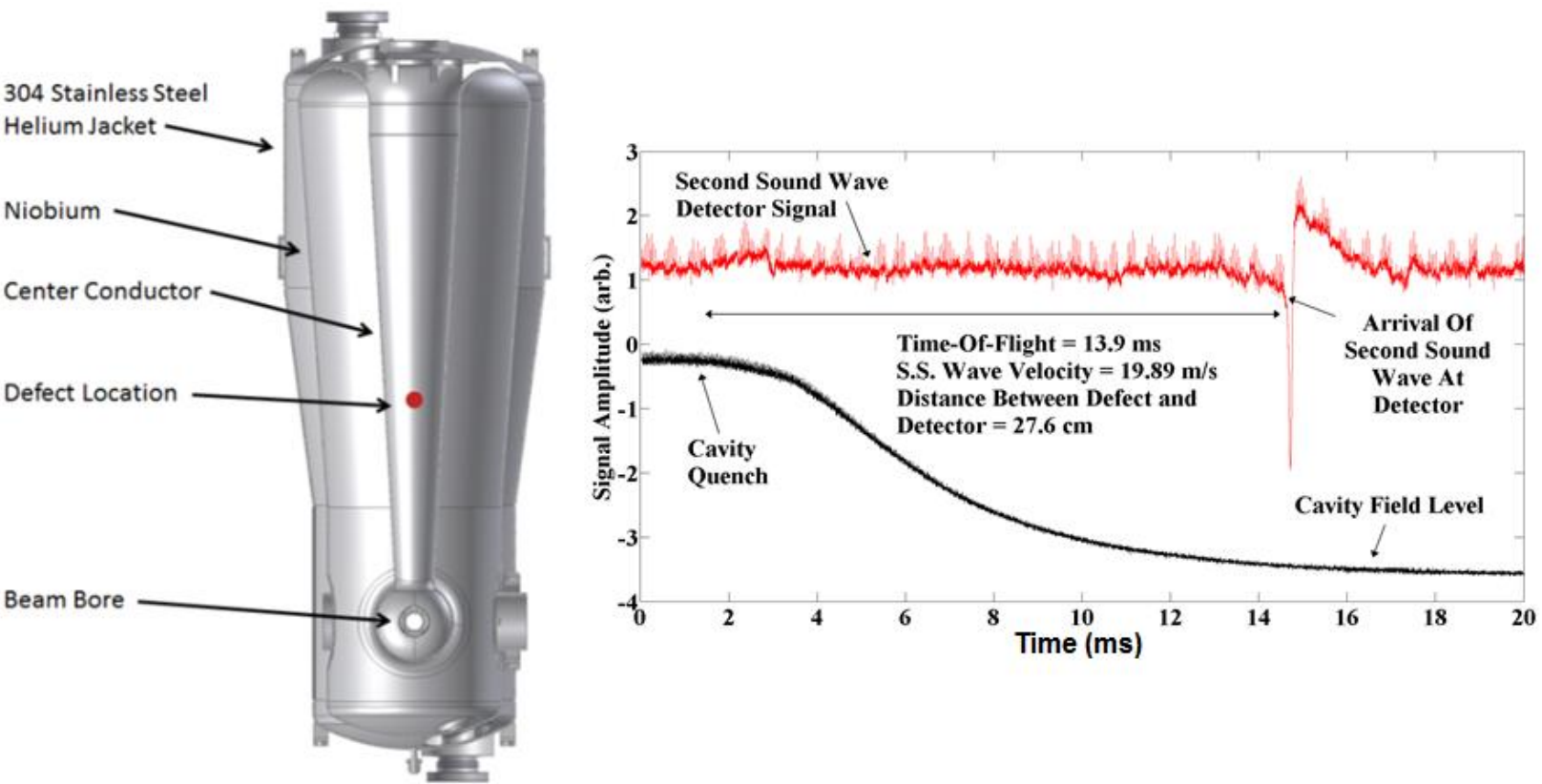

Figure 1: (Top) The cavity with a red dot placed at the height of the defect in the center conductor, the cavity is 53 inches from top to bottom. (Bottom) A single quench event with an oscillating superleak transducer signal and the cavity transmitted power. 


\subsection{Cold Test Results}

The DTRA QWR cavity vacuum was allowed to pump for 72 hours reaching a pressure $<1$ e-7 torr prior to cooldown. The cavity was cooled to $4.2 \mathrm{~K}$ with care taken to limit the time spent between 50 and $165 \mathrm{~K}$ to about 45 minutes even with the hydrogen degassing. The cavity was conditioned using up to 10 watts of RF power to remove the low-level multipacting barriers over $\sim 2$ hours and then 5 minutes of pulsed power processing at $4.6 \mathrm{~K}$ with $4 \mathrm{~kW}$ of forward power. Following this conditioning the performance given in figure 3 was observed with no significant field emission. Note that the residual resistance of the cavity at low-fields is less than $4.5 \mathrm{n} \Omega$ at $4.6 \mathrm{~K}$ and $2.5 \mathrm{n} \Omega$ at $2 \mathrm{~K}$. The gradients presented in figure 3 were not limited by any fundamental phenomena; e.g., defect-initiated quench or field emission. The results were limited administratively to avoid exceeding the ANL limits on x-ray production. The cavity is only partially shielded behind a high-density concrete wall and is not in a complete cavelike enclosure. This allows considerable $\mathrm{x}$-ray shine to reach experimenters. To reduce the average $\mathrm{x}$-ray production the cavity was operated with a $1.6 \%$ duty cycle to safely comply with the ANL guidelines. The data points measured in this manner are highlighted in green and are the three highest gradient $2 \mathrm{~K}$ measured data points. Please note that the cavity dissipated power measurement accuracy decreased due to measuring these data points in the over-coupled limit but the relative accuracy of the field amplitude is unchanged. The highest data point measured was repeatable and corresponded to a voltage gain of 7.4 MV and peak surface fields of $165 \mathrm{mT}$ and $117 \mathrm{MV} / \mathrm{m}$. The best 9elliptical cell cavity built for the ILC program reached $175 \mathrm{mT}$ and $84 \mathrm{MV} / \mathrm{m}$. The quarter-wave cavity tested here reached comparable peak surface magnetic fields relative to the best 9-cell cavity and exceeds the peak surface electric field by $40 \%$ [1]. The previous best reduced beta cavity was a spoke cavity tested at FNAL which reached $127 \mathrm{mT}$ and $85 \mathrm{MV} / \mathrm{m}$ after extensive conditioning [3].

It is important to note that at no point was the QWR tested here quenched. The high field data points do not represent a fundamental limit on the cavity performance and further testing is required to determine the cavity fundamental limits. This limitation was due to Argonne's administrative limits on X-ray production and the available RF power being insufficient to excite the cavity with a shorter pulse. These limits may also be overcome by upgrading the radiation shielding of the Argonne SRF cavity test facility which was initially designed and build for much lower performance cavities.
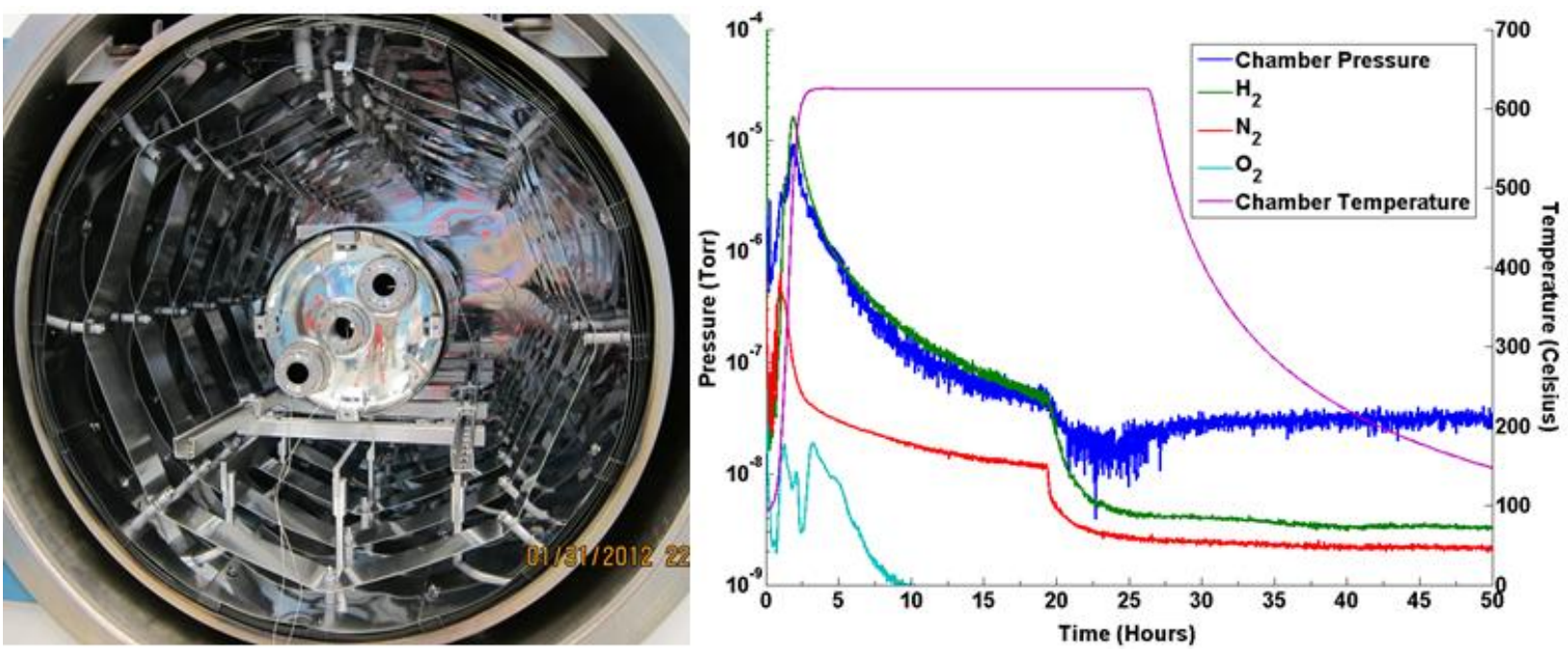

Figure 2: Left, end view of the quarter-wave cavity inside the Fermilab high-vacuum oven immediately before closing the door and starting the bake. Right, residual gas pressures of the dominant species and the cavity temperature during the bake. Notice that the cavity was baked at $625 \mathrm{C}$ for 24 hours. 


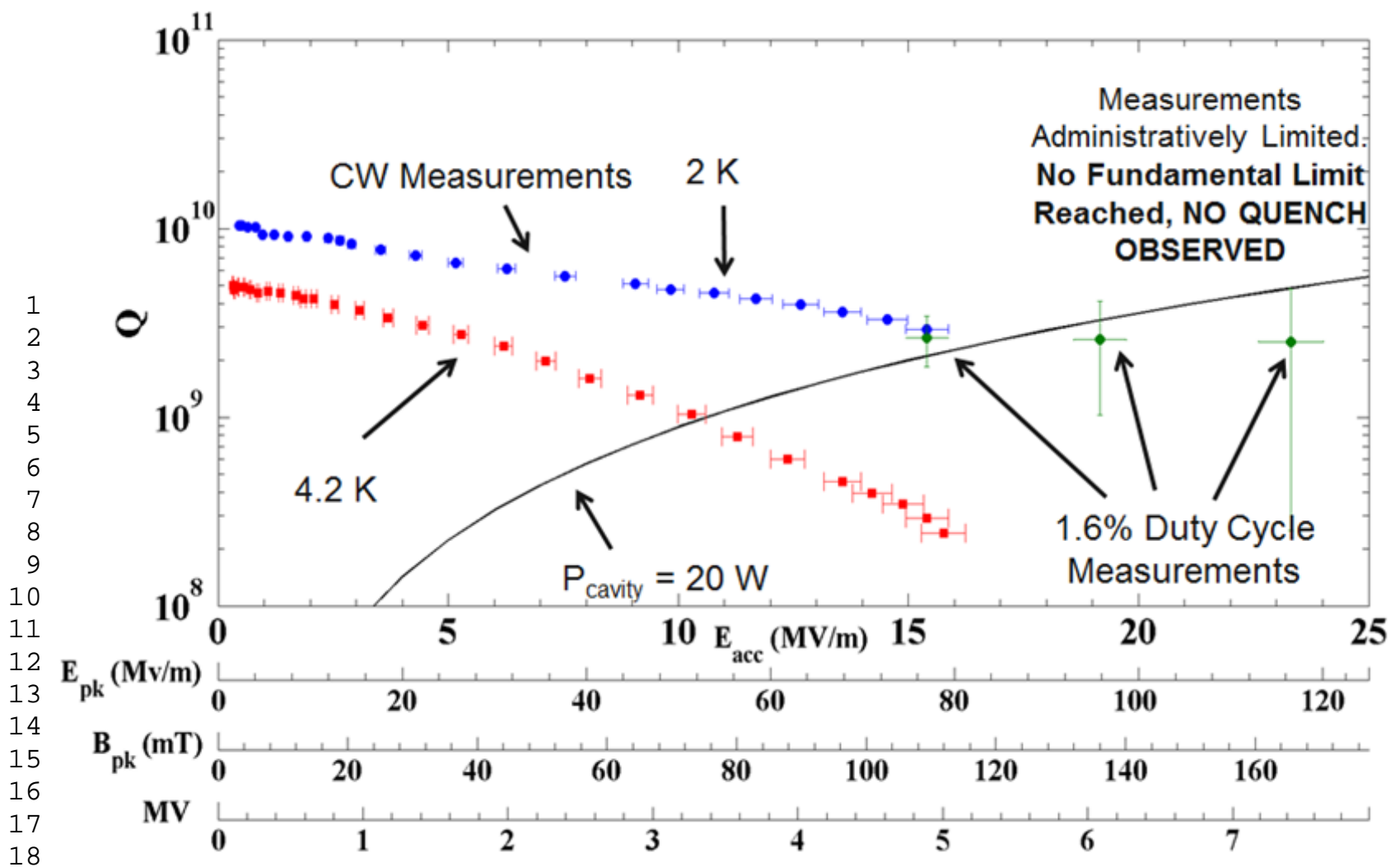

Figure 3: The measured performance of the QWR. There are 4 horizontal axes corresponding to the accelerating gradient, peak surface electric and magnetic fields, and the voltage gain of a synchronous $\beta=0.077$ ion. The vertical axis corresponds to the cavity quality factor. The blue data points were measured at $2 \mathrm{~K}$ and the red data

\section{Initial 4.5 K QWR Cryomodule Performance}

In total 8 QWR cavities were made. After the processing and test results presented in section 3 we changed our procedures to include:

- The hand sanding did not stop with 220 grit sandpaper. Finer grit sandpapers were used successively in the following steps: 120 grit, 220 grit, 320 grit, 400 grit and 600 grit. This produced a notably finer surface finish.

- The hydrogen degassing bake was shortened to 10 hours (less than half of the duration of the previous bake).

- The bulk electropolish procedure $\left(\sim 120 \mu \mathrm{m}\right.$ removal) temperature was increased to $30<\mathrm{T}<35^{0} \mathrm{C}$. The final light electropolish ( $20 \mu \mathrm{m}$ removal) temperature was also increased to $25<\mathrm{T}<30^{\circ} \mathrm{C}$.

With this set of parameters the remaining 6 QWR cavities were fabricated and several of them were tested off line prior to installation in the new ATLAS cryomodule. The cold test results for these cavities is shown in figure 4 . The initial prototype cavity and the DTRA QWR test results are included for completeness. Notice that all of the cavities exhibit extremely low residual resistances $\sim 1 \mathrm{n} \Omega$ or less and far exceed the ATLAS goal of operating at $8 \mathrm{MV} / \mathrm{m}$ at $4.5 \mathrm{~K}$. Cryomodule operations in support of the ATLAS.national user facility started in March 2014. In the short time the cryomodule has operated with real-estate gradients exceeding $3.3 \mathrm{MV} / \mathrm{m}$ and a total cryogenic load of $46 \mathrm{~W}$, with no fundamental limit restricting this operating level to higher values.

This cryomodule operating with real-estate gradients $>3.3 \mathrm{MV} / \mathrm{m}$ while phase locked to ATLAS beams is a significant improvement over current state for the art; previous best is $\sim 1.1 \mathrm{MV} / \mathrm{m}$ at TRIUMF for $\beta=0.071$ [5] which have been in highly reliable operation for many years. This represents not only a major step forward for the 7 


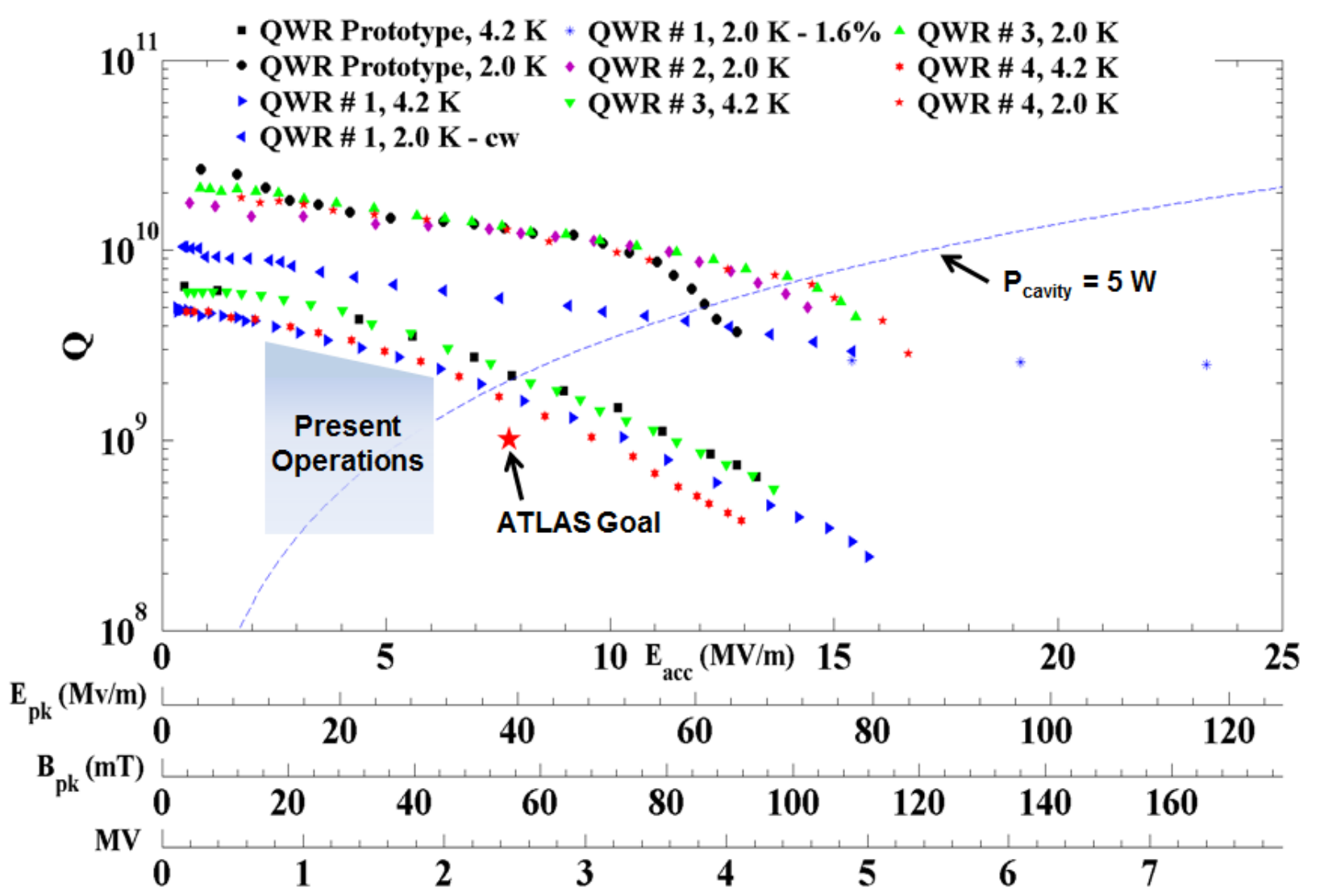

Figure 4: Q-curves for 5 different $72 \mathrm{MHz}$ quarter-wave cavities. The dashed curve is a line of constant, 5W, power dissipated in the cavity. QWR \# 1 could not be quenched due to ANL administrative limits on radiation production, and the cavities fabricated after QWR1 all quenched above $17 \mathrm{MV} / \mathrm{m}$.

field but also demonstrates the real possibility of operating low-beta cryomodules at even higher real-estate gradients.

\section{Impact and Future Applications}

The results presented here characterize the world's highest peak-field performance in a low-beta cavity designed for heavy-ion particle accelerators and the world's highest preforming cryomodule for heavy ion traveling with a $\beta$ 0.08 . The results are not fundamentally limited at this time and more effort is required to determine the maximum performance of the existing cavities and cryomodule. These results are encouraging for future projects and are an essential step toward proposing low-beta accelerators with higher baseline accelerating gradients which both shorten and reduce the cost of these installations.

\section{Acknowledgements}

This material is based upon work supported by the U.S. Department of Energy, Office of Science, Office of Nuclear Physics, under contract No. DE-AC02-06CH11357 and by the U.S. Defense Threat Reduction Agency. This research used resources of ANL's ATLAS facility, which is a DOE Office of Science User Facility.

\section{References}

[1] Geng, R.L., 2011, "Overview of ILC High Gradient Cavity R\&D at Jefferson Lab," In the Proceedings of the 2011 Conference on RF Superconductivity, 25-29 July 2011, Chicago, Illinois, USA, Pg. 74 (2012). 
[2] J. Popielarski et al, "Dewar Testing of beta $=0.085$ Quarter-Wave Resonators at MSU," In the Proceedings of the 2011 Conference on RF Superconductivity, 25-29 July 2011, Chicago, Illinois, USA, Pg. 537 (2012).

[3] L. Ristori et al, "Design, Fabrication and Testing of Single Spoke Resonators at Fermilab," In the Proceedings of the 2009 Conference on RF Superconductivity, 20-25 September 2009, Berlin, Germany, Pg. 550 (2010).

[4] P.N. Ostroumov et al, "Upgrade of Argonne's CW SC Heavy Ion Accelerator," In the Proceedings of the 2013 North American Particle Accelerator Conference, 29 September - 4 October 2013, Pasadena, California, Pg. 737 (2013).

[5] R.E. Laxdall et al, "ISAC-II: Status of the 20 MV upgrade, “ In the Proceedings of the 2009 Conference on RF Superconductivity, 20-25 September 2009, Berlin, Germany, Pg., 81.

[6] M.Lindroos et al, "The ESS Linac Design," In the Proceedings of the 2012 Linear Accelerator Conference, 9-14 September 2012, Tel Aviv, Israel, Pg. 768 (2013).

[7] R. Ferdinand et al, "Status and Challenges of Spiral 2 SRF Linac," In the Proceedings of the 2013 International Conference on RF Superconductivity, 23-27 September 2013, Paris, France, Pg. 11 (2012).

[8] B.H. Kang et al, "ISOL Facility for Rare Isotope Beams at RAON," J. Korean Phys. Soc. 63, Pg. 1473 (2013).

3 [9] Li Han et al, "Development of a $325 \mathrm{MHz}$ beta $=0.12$ superconducting single spoke cavity for China-ADS," Chinese Phys. C 38, Pg. 077008 (2014).

10] F. Orsini et al, "Vertical Tests Results of the IFMIF Cavity Prototypes and Cryomodule Development," In the Proceedings of the 2011 Conference on RF Superconductivity, 25-29 July 2011, Chicago, Illinois, USA, Pg. 667 (2012).

6 [11] S. Nagaitsev et al, "PXIE: Project X Injector Experiment," In the Proceedings of the 2012 International Particle Accelerator Conference, 2025 May 2012, New Orleans, Louisiana, Pg. 3874 (2013).

[12] J. Rodnizski et al, "SARAF Phase II P/D 40 MeV Linac Design Studies," In the Proceedings of the 2012 Linear Accelerator Conference, 9 14 September 2012, Tel Aviv, Israel, Pg. 100 (2013).

[13] J. Wei et al, “The Very High Intensity Future," In the Proceedings of the 2012 Linear Accelerator Conference, 9-14 September 2012, Tel Aviv, Israel, Pg. 17 (2013).

[14] D. MicGinnis, "New Design Approaches for High Intensity Superconducting Linacs - The New ESS Linac Design," In the Proceedings of the 2014 International Particle Accelerator Conference, 15-20 June 2014, Dresden, Germany, Pg. 23 (2014).

[15] V. Yakovlev and C. Ginsburg, "SRF Linac Technology Development at Fermilab," In the Proceedings of the 2012 Linear Accelerator Conference, 9-14 September 2012, Tel Aviv, Israel, Pg. 110 (2013).

[16] D. Longuevergne et al, "Performance Limitations Studies on ISAC-II QWR's and E-Linac Elliptical Cavities at Triumf," In the Proceedings of the 2011 Conference on RF Superconductivity, 25-29 July 2011, Chicago, Illinois, USA, Pg. 97 (2012).

[17] P.-E. Bernaudin et al, "Status of the SPIRAL 2 Superconducting Linac," In the Proceedings of the 2010 International Particle Accelerator Conference, 23-28 May 2010, Kyoto, Japan, Pg. 732 (2010).

[18] Kelly, M.P., Conway, Z.A., Gerbick, S.M., Kedzie, M.J., Murphy, R.C., Mustapha, B., Ostroumov, P.N., and Reid, T.C., 2011, "SRF Advances for ATLAS and Other Beta<1 Applications," In the Proceedings of the 2011 Conference of RF Superconductivity, 25-29 July 2011, Chicago, Illinois, USA, Pg. 680 (2011).

[19] Ostroumov, P.N., Fuerst, J.D., Kelly, M.P., Mustapha, B., Shepard, K.W., and Xu, J., 2009, "A New ATLAS Efficiency and Intensity Upgrade Project," In the Proceedings of the 2009 Conference on RF Superconductivity, 20-25 September 2009, Berlin, Germany, Pg. 218 (2010).

[20] Conway, Z.A., Hartill, D.L., Padamsee, H.S., and Smith, E.N., 2009, "Defect Location in Superconducting Cavities Cooled with He-II Using Oscillating Superleak Transducers," In the Proceedings of the 2009 Particle Accelerator Conference, 4-8 May 2009, Vancouver, British Columbia, Canada, Pg. 921 (2009). 